Author affiliations appear at the end of this article.

Published at ascopubs.org/journal/jco on December 27, 2016.

Support information appears at the end of this article.

Corresponding author: Iris D. Nagtegaal, MD, Department of Pathology 824

Radboud University Medical Center, PO Box 9101, 6500 HB, Nijmegen, the Netherlands; e-mail: iris.nagtegaal@ radboudumc.nl.

() 2016 by American Society of Clinical Oncology

0732-183X/16/3499-1/\$20.00

\title{
Tumor Deposits in Colorectal Cancer: Improving the Value of Modern Staging-A Systematic Review and Meta-Analysis
}

Iris D. Nagtegaal, Nikki Knijn, Niek Hugen, Helen C. Marshall, Kenichi Sugihara, Tibor Tot, Hideki Ueno, and Philip Quirke

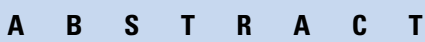

\section{Purpose}

Colorectal cancer (CRC) treatment is largely determined by tumor stage. Despite improvements made in the treatment of various types of metastatic disease, staging has not been refined. The role of tumor deposits (TDs) in staging remains debated. We have assessed the relation of TDs with metastatic pattern to evaluate whether TDs might add significant new information to staging.

\section{Methods}

We performed a systematic literature search that was focused on the role of TDs in CRC. Studies with neoadjuvant-treated patients were excluded. Data on stage, histologic factors, and outcome were extracted. Data from four large cohorts were analyzed for the relevance of the presence of TDs, lymph node metastases (LNMs), and extramural vascular invasion (EMVI) on the pattern of metastases and outcomes.

\section{Results}

Of 10,106 included patients with CRC, $22 \%$ presented with TDs. TDs are invariably associated with poor outcome. Presence of TDs was associated with presence of LNMs and EMVI. In a pairwise comparison, effects of TD were stronger than those of both LNMs and EMVI. In the logistic regression model, TDs in combination with LNMs is the strongest predictor for liver (odds ratio [OR], 5.5), lung (OR, 4.3) and peritoneal metastases (OR, 7.0). Presence of EMVI adds information for liver and lung metastases, but not for peritoneal metastases.

\section{Conclusion}

We have shown that TDs are not equal to LNMs or EMVI with respect to biology and outcome. We lose valuable prognostic information by allocating TDs into nodal category N1c and only considering TDs in the absence of LNMs. Therefore, we propose that the number of TDs should be added to the number of LNMs to derive a final $\mathrm{N}$ stage.

\section{J Clin Oncol 34. (C) 2016 by American Society of Clinical Oncology}

\section{INTRODUCTION}

Staging of cancer is one of the cornerstones of cancer treatment. The TNM staging system is an anatomically based classification that is applied worldwide for many tumor types. Originally, this system was used to determine prognostic outcomes and to enable the international comparison of different cohorts. With increasing treatment possibilities, tumor stage has become one of the main selection criteria for (adjuvant) therapy. In colorectal cancer (CRC), stage III patients are generally treated with systemic adjuvant therapy, as are patients with high-risk stage II disease. ${ }^{1,2}$

However, for many patients with metastatic disease, cytotoxic therapy is no longer their only treatment option and more widespread multimodality treatment with curative intent has become possible. Patients with oligometastases in liver or lung can undergo curative treatment in ever increasing numbers, ${ }^{3,4}$ and patients with peritoneal disease can undergo cytoreduction with hyperthermic intraperitoneal chemotherapy treatment. ${ }^{5}$ Clinical trials that will investigate treatment with adjuvant hyperthermic intraperitoneal chemotherapy in high-risk patients are currently recruiting. ${ }^{6}$ Therefore, we need more detailed staging systems that enable a better estimation for recurrence risk at different sites to guide new treatment choices.

In recent editions of the TNM staging system, inclusion of tumor deposits (TDs) within nodal staging has given rise to worldwide discussions. ${ }^{7-12}$ Other important prognostic 
features, such as extramural vascular invasion (EMVI), are acknowledged but not included in staging. One may wonder whether we lose useful information by ignoring the former and placing TDs with different etiologies into the nodal category, N1c, only in the absence of lymph node metastases (LNMs). If TDs are equal to LNMs, both in prognostic and biologic sense, this would simplify the staging systems as they can be placed in the $\mathrm{N}$ category without loss of information; however, if TDs add information to staging, either alone or taking into account their etiology, we should apply specific substaging.

We assessed the prognostic impact of TDs by performing a systematic review of existing data, investigated the association of TDs with other histologic prognostic factors, and determined whether TD status influenced the metastatic pattern in CRC. On the basis of the results, we propose revisions to be considered for the modern anatomic staging of CRC.

\section{METHODS}

\section{Strategy for Search of Articles and Selection Criteria}

A comprehensive literature search for published studies was performed by using Embase and Medline databases (OvidSP software; Ovid Technologies, New York, NY) from inception to July 29, 2015 using the following keywords: "tumor deposits" or "microfoci" or "non-nodal" or "nodal independent" or "neoplastic foci" or "tumor aggregate" or "discontinuous" or "extranodal" or "staging" in combination with "Colorectal Neoplasms"[Mesh] "Cecal Neoplasms" [Mesh] or "colorectal" or "colon" or "rectum" or "rectal" and "cancer" or "carcinoma" or "tumor", limited by "Survival Analysis" [Mesh]. Additional searches were performed by manual cross-referencing.

Only original studies that were published in English with at least 100 patients were selected. In case of overlapping patient data, results of the largest study or of the study with longest follow-up were included in this meta-analysis. Studies in which histology was not reviewed for whole cohorts were excluded, as reporting on TDs without histologic review is unreliable and incomplete. Studies that included patients who were treated with neoadjuvant therapy were excluded. Test and validation cohorts that have been described in the individual studies are separately analyzed.

\section{Data Extraction}

For each study, the number of patients in both the TD-positive and TD-negative groups were obtained. Data on tumor stage, histologic factors, 5-year disease-free survival (DFS), 5-year disease-specific survival (DSS), and 5-year overall survival (OS) were extracted from all studies. Data were entered in SPSS for Windows version 22 (SPSS, Chicago, IL) and Review Manager (version 5.3; Cochrane Tech, London, United Kingdom). Data were retrieved by two independent investigators (I.D.N. and N.K.).

\section{Quality Assessment and Risk of Bias}

A scale to assess the quality of study reporting was developed on the basis of the REMARK guidelines and focused on TDs (Appendix Table A1, online only). ${ }^{13,14}$ All studies were subjected to quality assessment; studies that were only used for correlation of TDs with other factors were subjected to quality assessment in which outcome-specific items were left out. The association between the quality of reporting and the hazard ratio (HR) was analyzed with scatter plots and nonparametric correlation testing. Publication bias was assessed by symmetry in funnel plots.

\section{Cohort Description}

Data from four cohorts was further explored to determine the association between TDs and metastatic patterns. These cohorts have been extensively described elsewhere. ${ }^{9,15}$ In brief, the first cohort is the test cohort from the Japanese Society for Cancer of the Colon and Rectum, which included 1,716 patients with stage I to III CRC who underwent curative surgery between 1994 and 1998, with an average follow-up of 93 months. ${ }^{15}$ The validation cohort from the Japanese Society for Cancer of the Colon and Rectum included 2,242 patients with stage I to III CRC who underwent curative surgery between 1999 and 2003, with an average follow-up of 68 months. ${ }^{15}$ The UK cohort consists of 455 patients with stage I to IV CRC who were included in the Medical Research Council CLASICC trial between July 1996 and July 2002, with an average follow-up of 63 months. ${ }^{9}$ The Swedish cohort represents a consecutive case series from Falu Lasarett of 505 patients with stage I to IV CRC who underwent surgery between 1998 and 2000, with an average follow-up of 63 months. ${ }^{9}$ Histology from all cases was reviewed with special attention for TDs, as has been described before. ${ }^{9,15}$

\section{Statistical Analysis}

A meta-analysis was performed with all available studies on correlation in terms of risk ratios (RRs) with 95\% CI. Data of univariable and multivariable analyses were entered in terms of $\mathrm{HR}$ with $95 \%$ CI. If no HR was reported, it was calculated from the published data, ${ }^{16}$ but only in studies with data on minimum and maximum follow-up times. A random effects model with inverse variance weighting of studies was used. In this model, each study was given a weight that was equal to the inverse of the variance of the effect estimate and served to minimize the variance of the combined effect. Forest plots were used to demonstrate consistency of results. For effect size, Z-statistic was used (standardized mean difference). Heterogeneity was assessed by using a $\chi^{2}$ test for heterogeneity with a $P$ value of $<.10$ to show the presence of significant heterogeneity. Furthermore, we applied $\mathrm{I}^{2}$ statistic — percentage of variation across studies that is a result of heterogeneity rather than chance-in combination with

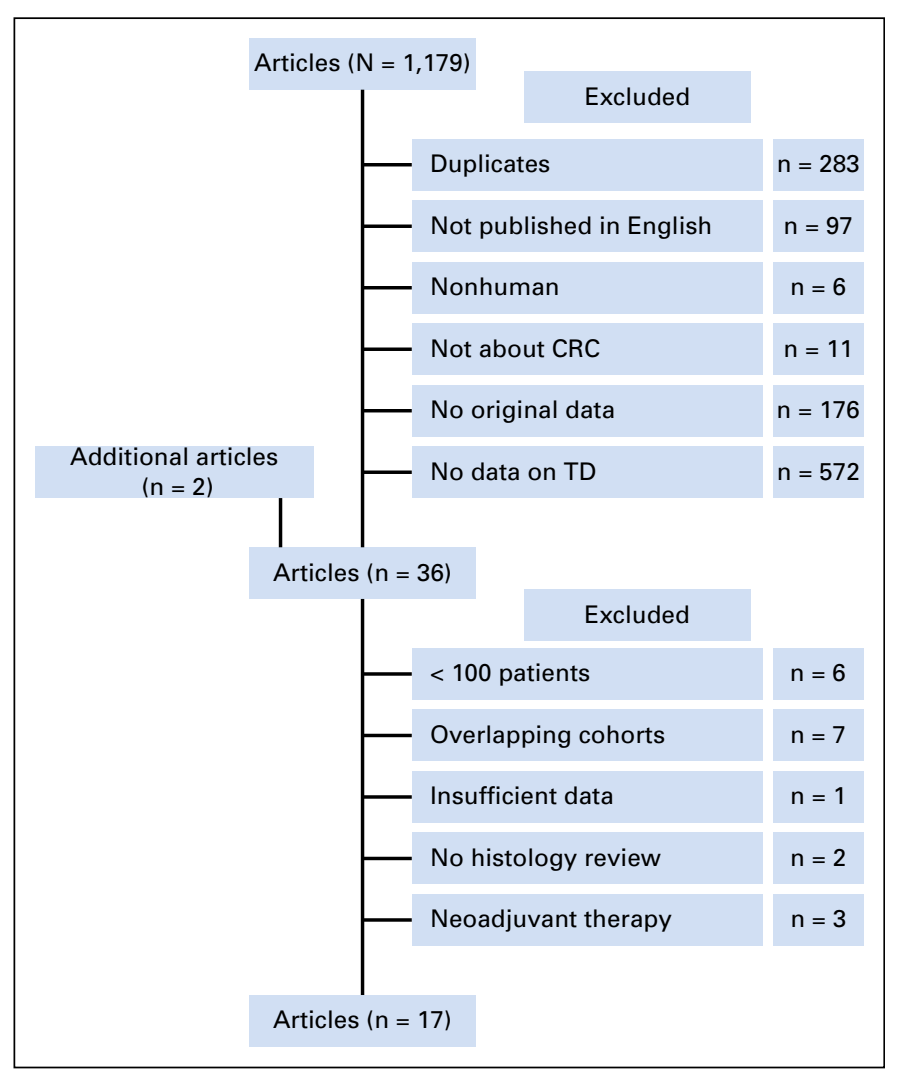

Fig 1. Flowchart of the article search strategy for systematic review. CRC, colorectal cancer; TD, tumor deposit. 
Tau-squared-estimate of between-study variance in a random effect meta-analysis. In case of heterogeneity, subanalyses for sample size, timeframe, and TNM stage were performed to identify the potential source of the heterogeneity. Logistic regression analysis was used to investigate the multivariable relationship of pathologic factors that predicted liver, lung, and peritoneal metastases in the four cohorts. In logistic regression analyses, the reference group used was the negative/negative group, that is, N0/TD negative. In the model, all first-order interactions were included, adjusting for cohort, LNMs, TDs, EMVI, and the combination of $\mathrm{LNM}^{\star} \mathrm{TD}, \mathrm{LNM}^{\star} \mathrm{EMVI}$, and TD*EMVI. The model was simplified by leaving out nonstatistical interactions with a $P$ value of $>.10$. Results were reported as odds ratios (ORs) with $95 \%$ CI. A $P$ value $\leq .05$ was considered statistically significant. Hosmer and Lemeshow test for goodness of fit was used to evaluate logistic regression models. We applied the Holm method for stepdown Bonferroni correction of multiple testing for each factor.

\section{RESULTS}

\section{Search Results}

A total of 574 studies were retrieved by the Medline database search, and 605 we found by using Embase. Duplicates were excluded $(n=283)$. A further 862 studies were excluded because they did not meet general inclusion criteria (Fig 1). We added two additional papers that fulfilled eligibility criteria. ${ }^{17,18}$ The remaining 36 papers concerned TDs in CRC. We excluded six studies because of insufficient patient numbers, ${ }^{19-24}$ one gave insufficient data for analysis, ${ }^{25}$ two studies did not perform histologic revision of all historic cases, ${ }^{18,26}$ and seven studies had overlapping data. ${ }^{12,27-32}$ Three studies included neoadjuvant-treated patients. ${ }^{33-35}$
The remaining 17 studies, which comprised 10,106 patients, were included in the meta-analysis. The main characteristics of the studies are listed in Table 1.

\section{Quality of the Reporting of the Included Studies}

Studies were subjected to quality assessment (Appendix Table A1). Thirteen studies were used for meta-analysis with outcome, ${ }^{15,17,36,37,41-45,47-49}$ of which nine studies could also be used for correlation of TDs with other factors. ${ }^{15,17,41-44,47-49}$ Two additional studies had no data on outcome and were only used for correlation of TDs with other factors. ${ }^{9,46}$ Moreover, three studies that were identified in our systematic review provided insufficient data for meta-analysis. ${ }^{38-40}$ The mean percentage of items that were reported in studies with outcome data was $66.6 \%$ (range, $39 \%$ to $84 \%$ ). The mean percentage of items reported in studies with data for correlation was $71.6 \%$ (range, $50 \%$ to $82 \%$ ).

\section{Frequency and Impact of TDs}

The average frequency of TDs for all studies was $22.0 \%$ (range, $4.9 \%$ to $41.8 \%)$.

Data on the impact of TDs on DFS in univariable analysis was available from five studies, which included, in total, 1,246 patients. In the presence of TDs, DFS was significantly decreased (HR, 2.2; 95\% CI, 1.6 to 3.0; Fig 2A). Considerable heterogeneity was observed among studies $\left(\mathrm{I}^{2}=78 \%\right)$. With respect to the quality assessment of studies, the percentage of items reported ranged from $50 \%$ to $84 \%$, and this did not correlate with the magnitude of

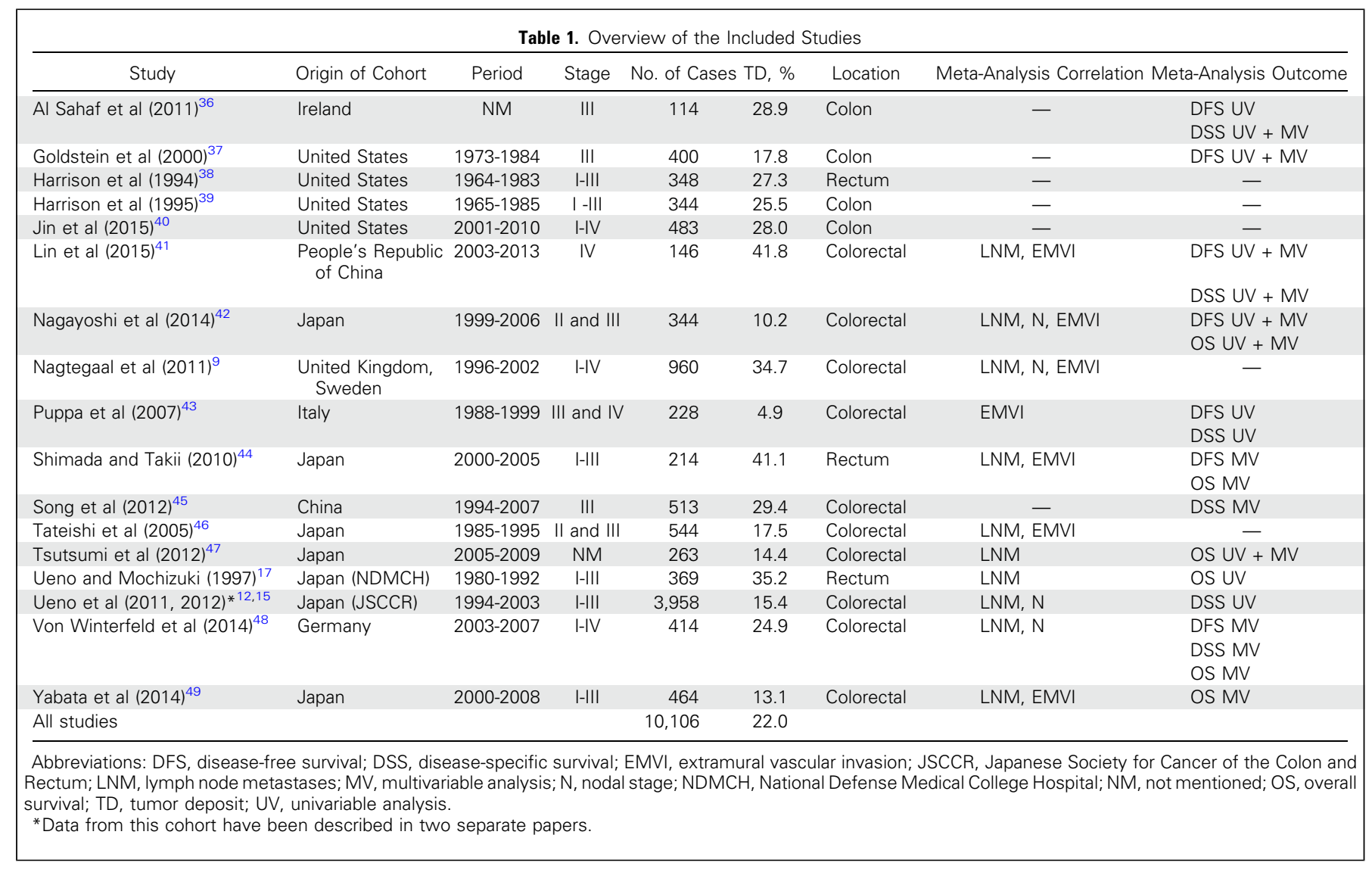




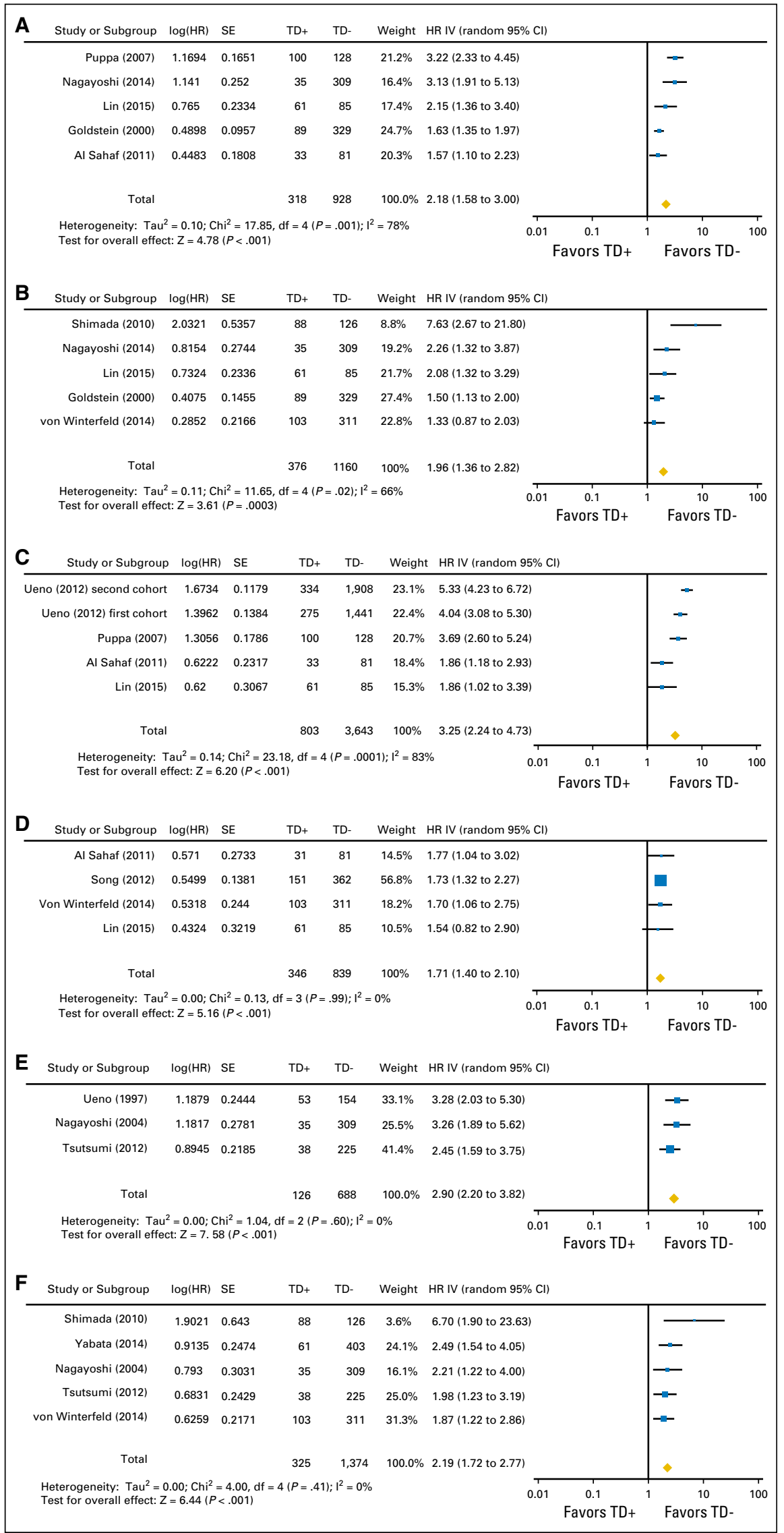

Fig 2. The impact of tumor deposits (TDs) on outcome. (A and B) Disease-free survival: univariable (A) and multivariable (B). (C and D) Disease-specific survival: univariable $(C)$ and multivariable (D). ( $E$ and $F$ ) Overall survival: univariable (E) and multivariable (F). HR, hazard ratio; SE, standard error; TD-, TD negative; TD +, TD positive. 
HR (Spearman $r=0.56 ; P=.35$ ). Multivariable DFS analysis was available in five studies that comprised 1,536 patients and that confirmed decreased DFS in the presence of TDs (HR, 2.0; 95\% CI, 1.4 to 2.8 ; Fig 2B). Substantial heterogeneity was observed among studies $\left(\mathrm{I}^{2}=66 \%\right)$. With respect to the quality assessment of the studies, the percentage of items reported ranged from $65 \%$ to $84 \%$, and this did not correlate with the magnitude of HR (Spearman $\mathrm{r}=0.82 ; P=.13)$.

The effect of TDs on DSS in univariable analysis was determined in five cohorts that comprised 4,446 patients (Fig 2C) and that confirmed decreased DSS in the presence of TDs (HR, 3.3; 95\% CI, 2.2 to 4.7 ). Considerable heterogeneity was observed among studies $\left(\mathrm{I}^{2}=83 \%\right)$. With respect to the quality assessment of the studies, the percentage of items reported ranged from $50 \%$ to $79 \%$, and this did not correlate with the magnitude of HR (Spearman $\mathrm{r}=-0.16 ; P=.78$ ). Multivariable DSS analysis was available in four studies that comprised 1,185 patients and that confirmed decreased DSS in the presence of TDs (HR, 1.7; 95\% CI, 1.4 to 2.1 ; Fig 2D). No heterogeneity was observed among studies $\left(\mathrm{I}^{2}=0 \%\right)$. The percentage of items reported ranged from $50 \%$ to $79 \%$, and this quality indicator did not correlate with the magnitude of HR (Spearman $\mathrm{r}=-0.80 ; P=.33$ ).

The impact of TDs on OS was available from three univariable and five multivariable cohorts with 814 and 1,699 patients, respectively (Figs $2 \mathrm{E}$ and $2 \mathrm{~F}$ ). OS was decreased in the presence of TDs (univariable HR, 2.9; 95\% CI, 2.2 to 3.8; and multivariable $\mathrm{HR}, 2.2 ; 95 \% \mathrm{CI}, 1.7$ to 2.8 ). No heterogeneity was observed in the univariable analysis $\left(\mathrm{I}^{2}=0 \%\right)$, nor the multivariable analysis $\left(\mathrm{I}^{2}=0 \%\right)$. For univariable studies, the percentage of items reported ranged from $56 \%$ to $84 \%$, which did not correlate with the magnitude of HR (Spearman $\mathrm{r}=-0.50$; $P=1.00$ ). For multivariable studies, the percentage ranged between $65 \%$ and $84 \%$, which did not correlate with the magnitude of HR (Spearman $\mathrm{r}=0.60 ; P=.35)$.

None of the analyses showed evidence of publication bias (Appendix Fig A1, online only). Observed heterogeneity in DFS and DSS analyses could not be explained by differences in sample size, timeframe, and TNM stage. Despite the observed heterogeneity, the direction of the effect in the forest plots is rather consistent. HR as a result of TD is smaller in the multivariable models, as would be expected because additional variance is accounted for; however, inclusion of these additional covariates does not diminish the significance of the HR as a result of TD. Additional information about the covariates that were included in the multivariable analyses are listed in Appendix Table A2 (online only).

\section{Subdivisions of TD: Does it Matter?}

The size of TDs influences prognosis: larger TDs $(>12 \mathrm{~mm}$ in diameter) have a significantly poorer DSS compared with small TD ( $\leq 3 \mathrm{~mm}$; HR, 2.5 and 3.2, respectively). ${ }^{15}$ Between $3 \mathrm{~mm}$ and $12 \mathrm{~mm}$, there was a nonsignificant increase in HR as a function of TD size. In another study ${ }^{44}$ small TDs, defined as $<2 \mathrm{~mm}$, showed a good DFS compared with that of larger TDs.

The contour of TDs can be described as smooth or irregular. Two studies ${ }^{1,44}$ of 214 and 3,958 patients demonstrated a trend toward poorer outcomes in the irregular groups; however, no direct comparison was performed.
Increasing numbers of TDs are associated with poor outcome. In the absence of LNMs, four or more TDs were associated with a significantly shorter survival in a small group of patients ( $\mathrm{n}=17 ; 16.5$ months $v 32.5$ months; $P=.025) .{ }^{40}$ Goldstein and Turner $^{41}$ showed that, regardless of nodal status, the 5-year survival of patients with three or more TDs was significantly worse compared with patients with only one or two TDs $(2 \% \mathrm{v}$ 24\%; $P<.01)$.

\section{Associations Between TD and Histologic Risk Factors}

The relationship between nodal status and the presence of TD was studied in 13 cohorts with a total of 7,583 patients (Appendix Fig A2A, online only). TDs were present in $8.7 \%$ of patients without LNMs compared with $41.6 \%$ of patients with LNMs. There were six cohorts in which the number of involved lymph nodes was studied (Appendix Fig A2B); there was a significant increase in TDs with increasing $\mathrm{N}$ stage in all studies $(P=.002$, Friedman test). RR for TDs in the presence of LNMs was 4.2 (95\% CI, 3.2 to 5.6; Appendix Fig A2A).

The relationship between TD and EMVI (as determined by examination of hematoxylin-eosin-stained slides) was studied in nine cohorts with a total number of 2,805 patients (Appendix Fig A2C). TDs were present in $20.9 \%$ of patients without EMVI compared with $31.6 \%$ of patients with EMVI. RR for TDs in the presence of EMVI was 2.6 (95\% CI, 1.8 to 3.7; Appendix Fig $\mathrm{A} 2 \mathrm{C})$.

\section{Comparison of LNMs, TDs, and EMVI}

Two studies investigated the prognostic power of TDs in combination with LNMs. ${ }^{44,46}$ Whereas absence and presence of both TDs and LNMs was associated with the best and worst outcomes, respectively, both studies suggest that the presence of only TDs is associated with a worse outcome than the presence of only LNMs.

To establish the value of TDs, LNMs, and EMVI in modern staging, we analyzed original data from four large cohorts of studies $^{9,15}$ that were selected in this systematic review in correlation with metastatic patterns, including both synchronous and metachronous metastases. Three different metastatic patterns were distinguished: liver metastases, lung metastases, and peritoneal metastases. In the four cohorts, which had a total of 4,918 patients, there were 397 liver metastases, 268 lung metastases, and 92 peritoneal metastases. The distribution of metastases was different between the cohorts, with higher percentages of liver and peritoneal metastases in the Sweden cohort (Fig 3A).

For RR at different metastatic locations (Fig 3B), the effect of LNMs was similar to that of TDs; however, the combination of TDs and LNMs was associated with a significantly higher risk of liver metastases than LNMs alone. When TDs and EMVI were compared (Fig 3C), it was clear that the presence of TDs significantly increased RR of liver metastases (RR, 3.6; 95\% CI, 2.6 to $5.0 v \mathrm{RR}, 1.7 ; 95 \% \mathrm{CI}, 1.3$ to 2.3 ). RR of TDs was not different from the RR of TD and EMVI combined. For lung metastases, the combination of TDs and EMVI significantly increased RR compared with EMVI alone. When the impact of EMVI in combination with LNMs was compared, it was clear that addition 

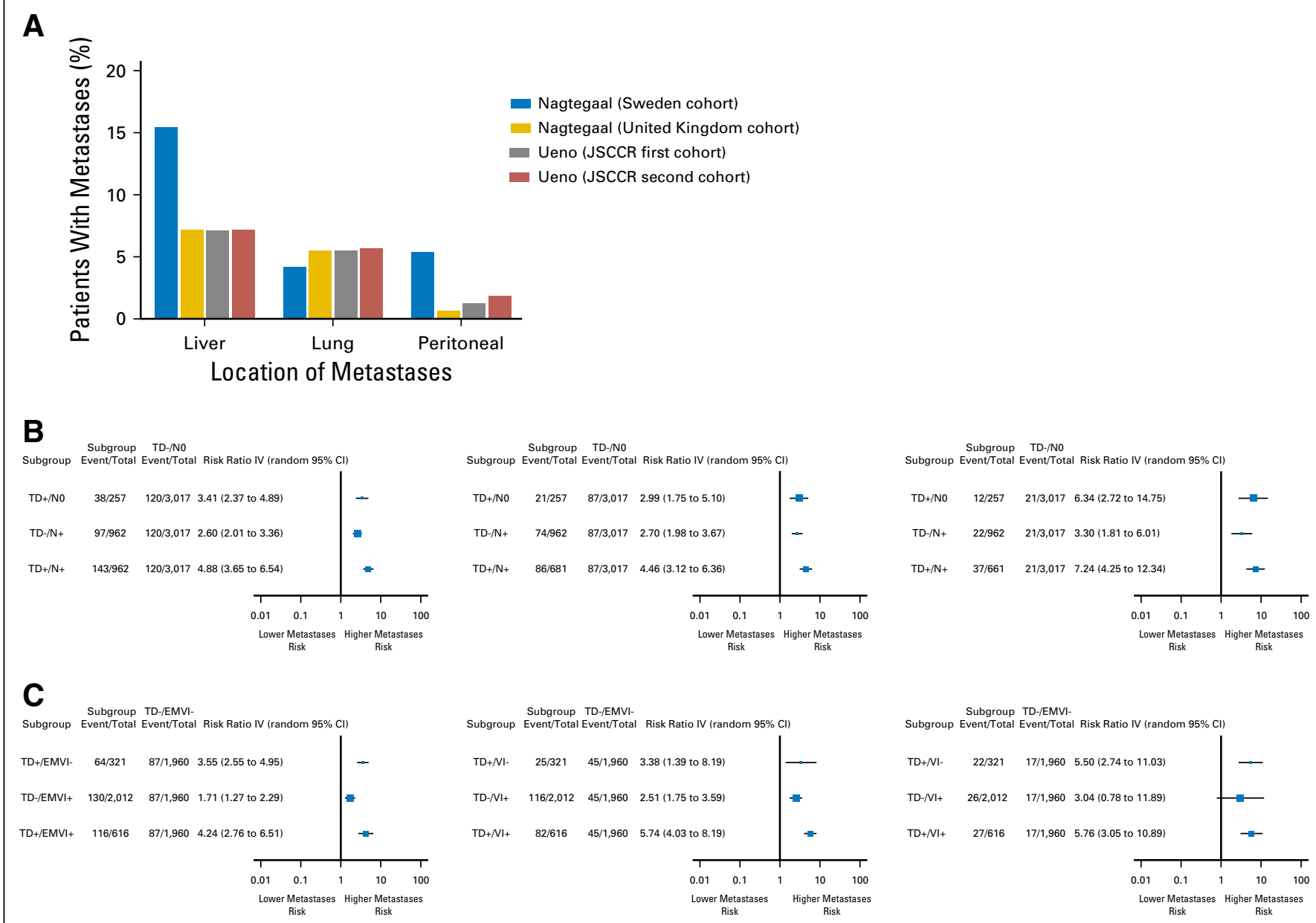

\section{D}
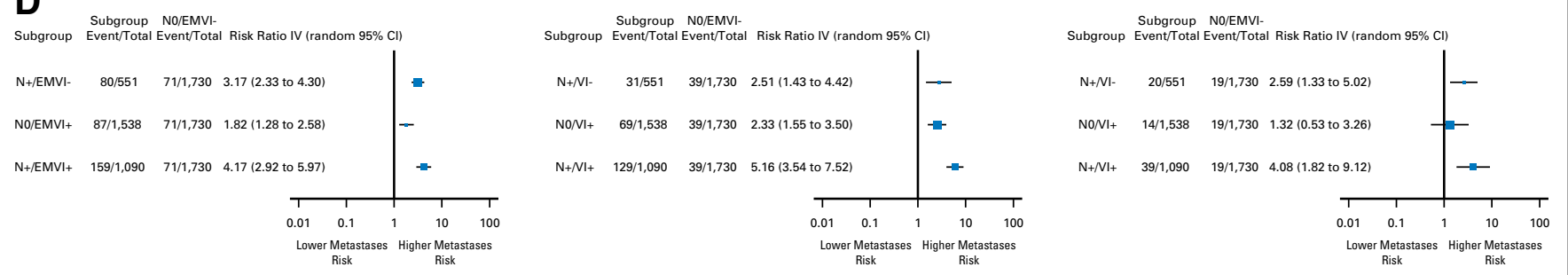

Fig 3. Metastatic patterns in relation to lymph node metastases (LNM), tumor deposit (TD), and extramural vascular invasion (EMVI) and combinations thereof. (A) Percentage of patients with different metastatic locations in the different cohorts. (B) Influence of LNM and TD on metastatic patterns. (C) Influence of EMVI and TD on metastatic patterns. (D) Influence of LNM and EMVI on metastatic patterns. According to the Japanese classification, no distinction between intramural vascular invasion and EMVI is made. ${ }^{50} \mathrm{EMVI}-$, no EMVI; EMVI+, EMVI present; JSCCR, Japanese Society for Cancer of the Colon and Rectum; NO, no LNM; N+, LNM positive; TD-, TD negative; TD+, TD positive.

of LNMs caused a higher RR for both lung and liver metastases (Fig 3D).

We subsequently evaluated the different factors by using a logistic regression model (Table 2). For liver metastases, TDs, LNMs, and EMVI were significant, with ORs of 3.6, 2.6, and 1.4, respectively. For lung metastases, the effects of TDs, LNMs, and EMVI were comparable (OR, 2.9, 2.5, and 2.0, respectively). For the development of peritoneal metastases, only TDs and LNMs contributed significantly (OR, 6.4 and 3.2, respectively), but not EMVI. Combination of TDs and LNMs did not increase the risk of peritoneal metastases compared with TDs alone.

\section{DISCUSSION}

In the current systematic review, we identified 17 large-scale studies that investigated the role of TDs in CRC. In a collection of 10,106 patients with CRC patients, the incidence of TDs was $22 \%$, which illustrates its potential value. The presence of TDs was invariably associated with a poorer outcome as illustrated by decreased DFS (HR, 1.7 to 2.0), DSS (HR, 1.7 to 3.9), and OS (HR, 2.2 to 2.9). Some unexplained heterogeneity was present in DFS and DSS analyses; however, OS analyses did not show heterogeneity. 


\begin{tabular}{|c|c|c|c|}
\hline Factor & Liver Metastases & Lung Metastases & Peritoneal Metastases \\
\hline No/TD- & 1.00 & 1.00 & 1.00 \\
\hline No/TD+ + & 3.57 (2.38 to 5.35$)$ & 2.86 (1.71 to 4.78$)$ & 6.44 (3.04 to 13.65 ) \\
\hline$N+/ T D-$ & 2.60 (1.96 to 3.44$)^{*}$ & 2.49 (1.81 to 3.44$) \dagger$ & $3.21(1.75$ to 5.90$) \ddagger$ \\
\hline $\mathrm{N}+/ \mathrm{TD}+$ & $5.54(4.23 \text { to } 7.25)^{*}$ & 4.29 (3.11 to 5.93$) \dagger$ & $6.97(3.96$ to 12.25$) \ddagger$ \\
\hline EMVI & 1.38 (1.08 to 1.77$)$ & 2.01 (1.48 to 2.72 ) & 1.25 (0.76 to 2.05$)$ \\
\hline Hosmer and Lemeshow goodness of fit & $P=.476$ & $P=.688$ & $P=.498$ \\
\hline
\end{tabular}

Recent editions of TNM have acknowledged the importance of TD by incorporating it in nodal staging. In the 5th edition of $\mathrm{TNM}^{51}$ the size of TD was considered important, but this was replaced by contour in the 6 th edition ${ }^{52}$ and by local interpretation in the 7th edition. ${ }^{53}$ Despite the clinical impact of these definitions, limited data are available to study both size and contour. Two studies ${ }^{15,44}$ have confirmed that size matters by demonstrating that larger TDs are associated with worse prognosis. Data on the impact of contour is less convincing.

The correlation between TDs and other types of regional spread might be part of the explanation of the poor prognosis. TDs occur more frequently in cases with perineural invasion $^{21,41,43}$ and lymphatic invasion. ${ }^{17,42-44,46,49}$ We summarized the most relevant correlations and demonstrated increased TDs in patients with LNMs and EMVI; however, data from multivariable studies still demonstrate an independent prognostic effect of TDs.

It is important to realize that TDs are not LNMs: the origin of TDs is diverse. By serial sectioning in a series of 30 irregular TDs, ${ }^{37}$ almost $40 \%$ showed a combined perineural, perivascular, and intravascular origin. A perineural origin was present in $77 \%$ of cases and an intravascular origin in $83 \%$ of cases. A similar setup with $69 \mathrm{TDs}^{54}$ showed similar diversity. Presence of vessels and nerves in the majority of TDs explains the worse prognosis of patients with TDs compared with that of patients with LNMs alone. Tumor access to more than one anatomic highway to metastatic locations creates more extensive tumor spread; therefore, we decided to evaluate the metastatic patterns that occur in the presence of TDs. The early study of Goldstein and Turner $^{37}$ suggested a significant impact of TDs in the development of intra-abdominal metastases. In their cohort, only $12 \%$ of patients without TDs developed peritoneal metastases compared with $44 \%$ of patients with TDs. In the current study, we examined original data from four different patient cohorts and the impact of TDs on the pattern of metastases. Presence of TDs and LNMs more than doubled the RR $(5.3 v 2.5)$ for liver metastases compared with LNM alone. Similar trends are observed for other metastatic patterns. A first explanation would be that TDs indeed reflect EMVI and thus explain the high risk of liver metastases ${ }^{55}$; however, when we compared TDs and EMVI, the difference was even more pronounced. Whether there is an unequivocal alternative biologic explanation ${ }^{56}$ remains to be investigated. From these results, it is clear that TDs do not equate to LNMs, nor recognizable EMVI, both in a prognostic and in a biologic sense. This study shows that by allocating all TDs into a nodal category, $\mathrm{pN} 1 \mathrm{c}$, and subsequently ignoring them in the presence of LNMs, valuable prognostic information is lost. The same argument can be made for EMVI; we also lose potential information on the likely sites for recurrence.

This study confirms that sufficient consistent evidence exists to now justify TD assessment in the management of CRC. However, there are a number of significant issues. The lack of definition in the current edition of TNM is not acceptable as it leads to poor interobserver agreement. ${ }^{57}$ True effects of the total number of TDs have not been determined, nor has this aspect been considered against the number of LNMs present. We do not know the optimal way to classify TDs after neoadjuvant treatment. Size of TDs seems to matter and further characterization is required. It is not clear how we should integrate these prognostic markers into the debate over when to use adjuvant therapy. ${ }^{58}$ Despite all of these issues, TDs and their number should be fully included in TNM staging. Inclusion of TDs only in the absence of LNMs is not justified by the evidence. TDs and their actual number should be considered equal to the number of LNMs in making treatment decisions; therefore, the number of TDs should be added to the number of LNMs in nodal staging to derive a final $\mathrm{N}$ stage.

\section{AUTHORS' DISCLOSURES OF POTENTIAL CONFLICTS OF INTEREST}

Disclosures provided by the authors are available with this article at ascopubs.org/journal/jco.

\section{AUTHOR CONTRIBUTIONS}

Conception and design: Iris D. Nagtegaal, Hideki Ueno, Philip Quirke Provision of study materials or patients: Kenichi Sugihara, Tibor Tot, Hideki Ueno, Philip Quirke

Collection and assembly of data: Iris D. Nagtegaal, Nikki Knijn, Helen C. Marshall, Kenichi Sugihara, Tibor Tot, Hideki Ueno

Data analysis and interpretation: Iris D. Nagtegaal, Nikki Knijn, Niek Hugen

Manuscript writing: All authors

Final approval of manuscript: All authors

Accountable for all aspects of the work: All authors 


\section{REFERENCES}

1. van de Velde CJ, Boelens PG, Borras JM, et al: EURECCA colorectal: Multidisciplinary management: European consensus conference colon \& rectum Eur J Cancer 50:1.e1-1.e34, 2014

2. Benson AB III, Venook AP, Bekaii-Saab T, et al: Colon cancer, version 3.2014. J Natl Compr Canc Netw 12:1028-1059, 2014

3. de Ridder JA, Lemmens VE, Overbeek LI, et al: Liver Resection for Metastatic Disease; A Population-Based Analysis of Trends. Dig Surg 33: 104-113, 2016

4. Booth CM, Nanji S, Wei X, et al: Outcomes of resected colorectal cancer lung metastases in routine clinical practice: A population-based study. Ann Surg Oncol 23:1057-1063, 2016

5. Elias D, Lefevre JH, Chevalier J, et al: Complete cytoreductive surgery plus intraperitoneal chemohyperthermia with oxaliplatin for peritoneal carcinomatosis of colorectal origin. J Clin Oncol 27: 681-685, 2009

6. Klaver CE, Musters GD, Bemelman WA, et al: Adjuvant hyperthermic intraperitoneal chemotherapy (HIPEC) in patients with colon cancer at high risk of peritoneal carcinomatosis; The COLOPEC randomized multicentre trial. BMC Cancer 15:428, 2015

7. Nagtegaal ID, Quirke P: Revised staging: Is it really better, or do we not know? J Clin Oncol 28: e397-e398; author reply e399-e400, 2010

8. Nagtegaal ID, Quirke P, Schmoll HJ: Has the new TNM classification for colorectal cancer improved care? Nat Rev Clin Oncol 9:119-123, 2011

9. Nagtegaal ID, Tot $T$, Jayne DG, et al: Lymph nodes, tumor deposits, and TNM: Are we getting better? J Clin Oncol 29:2487-2492, 2011

10. Quirke $P$, Williams GT, Ectors $N$, et al: The future of the TNM staging system in colorectal cancer: Time for a debate? Lancet Oncol 8:651-657, 2007

11. Ueno H, Mochizuki H, Shirouzu K, et al: Multicenter study for optimal categorization of extramural tumor deposits for colorectal cancer staging. Ann Surg 255:739-746, 2012

12. Ueno $H$, Mochizuki $H$, Akagi $Y$, et al: Optimal colorectal cancer staging criteria in TNM classification. J Clin Oncol 30:1519-1526, 2012

13. Knijn N, Simmer F, Nagtegaal ID: Recommendations for reporting histopathology studies: A proposal. Virchows Arch 466:611-615, 2015

14. McShane LM, Altman DG, Sauerbrei $W$, et al: Reporting recommendations for tumor marker prognostic studies. J Clin Oncol 23:9067-9072, 2005

15. Ueno H, Mochizuki H, Shirouzu K, et al: Actual status of distribution and prognostic impact of extramural discontinuous cancer spread in colorectal cancer. J Clin Oncol 29:2550-2556, 2011

16. Parmar MK, Torri V, Stewart L: Extracting summary statistics to perform meta-analyses of the published literature for survival endpoints. Stat Med 17:2815-2834, 1998

17. Ueno $\mathrm{H}$, Mochizuki $\mathrm{H}$ : Clinical significance of extrabowel skipped cancer infiltration in rectal cancer. Surg Today 27:617-622, 1997

18. Belt EJ, van Stijn MF, Bril H, et al: Lymph node negative colorectal cancers with isolated tumor deposits should be classified and treated as stage III. Ann Surg Oncol 17:3203-3211, 2010

19. Ishikawa K, Hashiguchi $Y$, Mochizuki $\mathrm{H}$, et al: Extranodal cancer deposit at the primary tumor site and the number of pulmonary lesions are useful prognostic factors after surgery for colorectal lung metastases. Dis Colon Rectum 46:629-636, 2003
20. Kiss L, Kiss R, Porr PJ, et al: Pathological evidence in support of total mesorectal excision in the management of rectal cancer. Chirurgia (Bucur) 106:347-352, 2011

21. Prabhudesai $A$, Arif $S$, Finlayson $C J$, et al: Impact of microscopic extranodal tumor deposits on the outcome of patients with rectal cancer. Dis Colon Rectum 46:1531-1537, 2003

22. Ratto C, Ricci R, Rossi $C$, et al: Mesorectal microfoci adversely affect the prognosis of patients with rectal cancer. Dis Colon Rectum 45:733-742 discussion 742-743, 2002

23. Tocchi A, Mazzoni G, Lepre L, et al: Total mesorectal excision and low rectal anastomosis for the treatment of rectal cancer and prevention of pelvic recurrences. Arch Surg 136:216-220, 2001

24. Ueno $H$, Mochizuki $H$, Fujimoto $H$, et al: Autonomic nerve plexus involvement and prognosis in patients with rectal cancer. Br J Surg 87:92-96, 2000

25. Prall $F$, Schmitt $O$, Schiffmann L: Tumor regression in rectal cancer after intensified neoadjuvant chemoradiation: A morphometric and clinicopathological study. World J Surg Oncol 13:155, 2015

26. Lo DS, Pollett A, Siu LL, et al: Prognostic significance of mesenteric tumor nodules in patients with stage III colorectal cancer. Cancer 112:50-54 2008

27. Lin $\mathrm{Q}, \mathrm{Ye} \mathrm{Q}$, Zhu D, et al: Determinants of longterm outcome in patients undergoing simultaneous resection of synchronous colorectal liver metastases. PLoS One 9:e105747, 2014

28. Puppa G, Ueno H, Kayahara M, et al: Tumor deposits are encountered in advanced colorectal cancer and other adenocarcinomas: An expanded classification with implications for colorectal cancer staging system including a unifying concept of intransit metastases. Mod Pathol 22:410-415, 2009

29. Tong $L L$, Gao $P$, Wang $Z N$, et al: Is the seventh edition of the UICC/AJCC TNM staging system reasonable for patients with tumor deposits in colorectal cancer? Ann Surg 255:208-213, 2012

30. Ueno $H$, Hashiguchi $Y$, Shimazaki $H$, et al: Peritumoral deposits as an adverse prognostic indicator of colorectal cancer. Am J Surg 207:70-77, 2014

31. Ueno $H$, Mochizuki $H$, Hashiguchi $Y$, et al: Extramural cancer deposits without nodal structure in colorectal cancer: Optimal categorization for prognostic staging. Am J Clin Pathol 127:287-294, 2007

32. Ueno $H$, Mochizuki $H$, Tamakuma S: Prognostic significance of extranodal microscopic foci discontinuous with primary lesion in rectal cancer. Dis Colon Rectum 41:55-61, 1998

33. Gopal P, Lu P, Ayers GD, et al: Tumor deposits in rectal adenocarcinoma after neoadjuvant chemoradiation are associated with poor prognosis. Mod Pathol 27:1281-1287, 2014

34. Song JS, Chang HJ, Kim DY, et al: Is the N1c category of the new American Joint Committee on cancer staging system applicable to patients with rectal cancer who receive preoperative chemoradiotherapy? Cancer 117:3917-3924, 2011

35. Swellengrebel HA, Bosch SL, Cats $A$, et al: Tumour regression grading after chemoradiotherapy for locally advanced rectal cancer: A near pathologic complete response does not translate into good clinical outcome. Radiother Oncol 112:44-51, 2014

36. Al Sahaf O, Myers E, Jawad M, et al: The prognostic significance of extramural deposits and extracapsular lymph node invasion in colon cancer. Dis Colon Rectum 54:982-988, 2011

37. Goldstein NS, Turner JR: Pericolonic tumor deposits in patients with $\mathrm{T} 3 \mathrm{~N}+\mathrm{MO}$ colon adenocarcinomas:
Markers of reduced disease free survival and intraabdominal metastases and their implications for TNM classification. Cancer 88:2228-2238, 2000

38. Harrison JC, Dean PJ, el-Zeky F, et al: From Dukes through Jass: Pathological prognostic indicators in rectal cancer. Hum Pathol 25:498-505, 1994

39. Harrison JC, Dean PJ, el-Zeky F, et al: Impact of the Crohn's-like lymphoid reaction on staging of right-sided colon cancer: Results of multivariate analysis. Hum Pathol 26:31-38, 1995

40. Jin $M$, Roth R, Rock JB, et al: The impact of tumor deposits on colonic adenocarcinoma AJCC TNM staging and outcome. Am J Surg Pathol 39: 109-115, 2015

41. Lin $\mathrm{Q}$, Wei $Y$, Ren $L$, et al: Tumor deposit is a poor prognostic indicator in patients who underwent simultaneous resection for synchronous colorectal liver metastases. Onco Targets Ther 8: 233-240, 2015

42. Nagayoshi K, Ueki T, Nishioka Y, et al: Tumor deposit is a poor prognostic indicator for patients who have stage II and III colorectal cancer with fewer than 4 lymph node metastases but not for those with 4 or more. Dis Colon Rectum 57:467-474, 2014

43. Puppa G, Maisonneuve $P$, Sonzogni $A$, et al: Pathological assessment of pericolonic tumor deposits in advanced colonic carcinoma: Relevance to prognosis and tumor staging. Mod Pathol 20: 843-855, 2007

44. Shimada Y, Takii Y: Clinical impact of mesorectal extranodal cancer tissue in rectal cancer: Detailed pathological assessment using whole-mount sections. Dis Colon Rectum 53:771-778, 2010

45. Song YX, Gao P, Wang ZN, et al: Can the tumor deposits be counted as metastatic lymph nodes in the UICC TNM staging system for colorectal cancer? PLoS One 7:e34087, 2012

46. Tateishi S, Arima S, Futami K, et al: A clinicopathological investigation of "tumor nodules" in colorectal cancer. Surg Today 35:377-384, 2005

47. Tsutsumi $S$, Watanabe $R$, Tabe $Y$, et al: Extranodal metastasis predicts poor survival in advanced colorectal cancer. Hepatogastroenterology 59:372-374, 2012

48. von Winterfeld $M$, Hoffmeister $M$, IngoldHeppner B, et al: Frequency of therapy-relevant staging shifts in colorectal cancer through the introduction of pN1c in the 7th TNM edition. Eur $J$ Cancer 50:2958-2965, 2014

49. Yabata $\mathrm{E}$, Udagawa $\mathrm{M}$, Okamoto $\mathrm{H}$ : Effect of tumor deposits on overall survival in colorectal cance patients with regional lymph node metastases. J Rural Med 9:20-26, 2014

50. Japanese Society for Cancer of the Colon and Rectum: Japanese Classification of Colorectal Carcinoma (ed 2). Tokyo, Japan, Kanehara, 2009

51. Sobin LH, Wittekind C: UICC TNM Classification of Malignant Tumours (ed 5). New York, NY, John Wiley \& Sons, 1997

52. Sobin LH, Wittekind C: TNM Classification of Malignant Tumours (ed 6). New York, NY, Wiley-Liss, 2002

53. Sobin LH, Gospodarowicz M, Wittekind C: TNM Classification of Malignant Tumours (ed 7). Circhester, United Kingdom, John Wiley \& Sons, 2009

54. Wünsch K, Müller J, Jähnig H, et al: Shape is not associated with the origin of pericolonic tumor deposits. Am J Clin Pathol 133:388-394, 2010

55. Talbot IC, Ritchie S, Leighton $\mathrm{MH}$, et al: The clinical significance of invasion of veins by rectal cancer. Br J Surg 67:439-442, 1980 
56. Fan XJ, Wan XB, Yang ZL, et al: Snail promotes lymph node metastasis and Twist enhances tumor deposit formation through epithelial-mesenchymal transition in colorectal cancer. Hum Pathol 44:173-180, 2013
57. Rock JB, Washington MK, Adsay NV, et al: Debating deposits: An interobserver variability study of lymph nodes and pericolonic tumor deposits in colonic adenocarcinoma. Arch Pathol Lab Med 138:636-642, 2014
58. Påhlman LA, Hohenberger WM, Matzel K, et al: Should the benefit of adjuvant chemotherapy in colon cancer be re-evaluated? J Clin Oncol 34: 1297-1299, 2016

\section{Affiliations}

Iris D. Nagtegaal, Nikki Knijn, and Niek Hugen, Radboud University Medical Center, Nijmegen, the Netherlands; Helen C. Marshall and Philip Quirke, Leeds University, Leeds, United Kingdom; Kenichi Sugihara, Tokyo Medical and Dental University, Tokyo; Hideki Ueno, National Defence Medical College, Tokorozawa, Japan; and Tibor Tot, Falu Lasarett, Falun, Sweden.

\section{Support}

Supported by Yorkshire Cancer Research and NIHR senior investigator (P.Q.). The digital pathology was supported by a Medical Research Council grant to the Leeds Institute of Data Analytics. 
Tumor Deposits in Colorectal Cancer: Improving the Value of Modern Staging—A Systematic Review and Meta-Analysis

The following represents disclosure information provided by authors of this manuscript. All relationships are considered compensated. Relationships are self-held unless noted. I = Immediate Family Member, Inst = My Institution. Relationships may not relate to the subject matter of this manuscript. For more information about ASCO's conflict of interest policy, please refer to www.asco.org/rwc or ascopubs.org/jco/site/ifc.

Iris D. Nagtegaal

Travel, Accommodations, Expenses: Amgen, Sakura

Nikki Knijn

No relationship to disclose

Niek Hugen

No relationship to disclose

Helen C. Marshall

No relationship to disclose

Kenichi Sugihara

No relationship to disclose

\section{Tibor Tot}

No relationship to disclose

\section{Hideki Ueno}

No relationship to disclose

Philip Quirke

Honoraria: Eisai

Consulting or Advisory Role: Eisai, Roche

Research Funding: IntegraGen, Amgen, Roche, HalioDx, Leica Biosystems, Ventana Medical Systems, Covance 


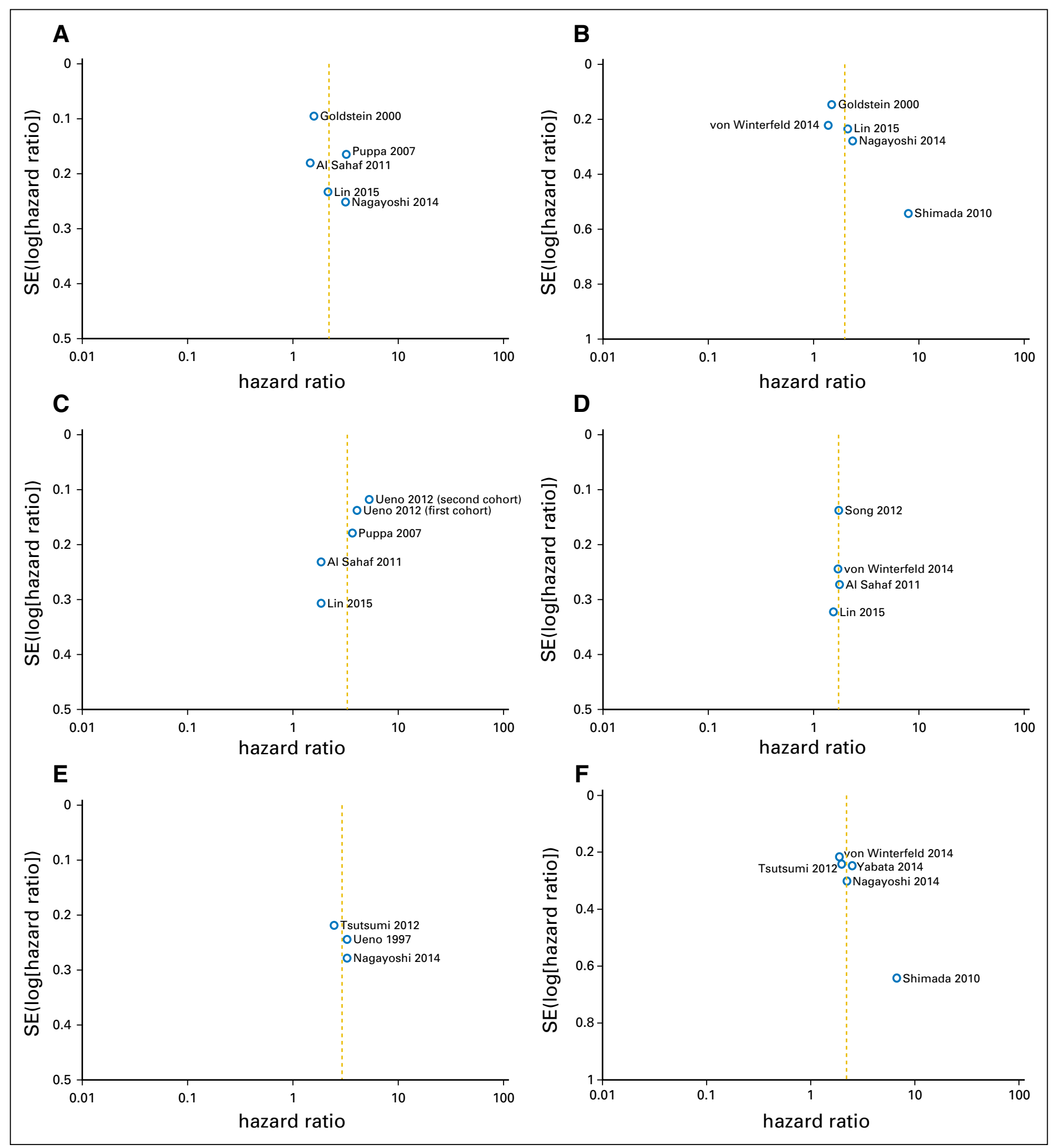

Fig A1. Funnel plots for the meta-analyses as shown in Fig 2. 


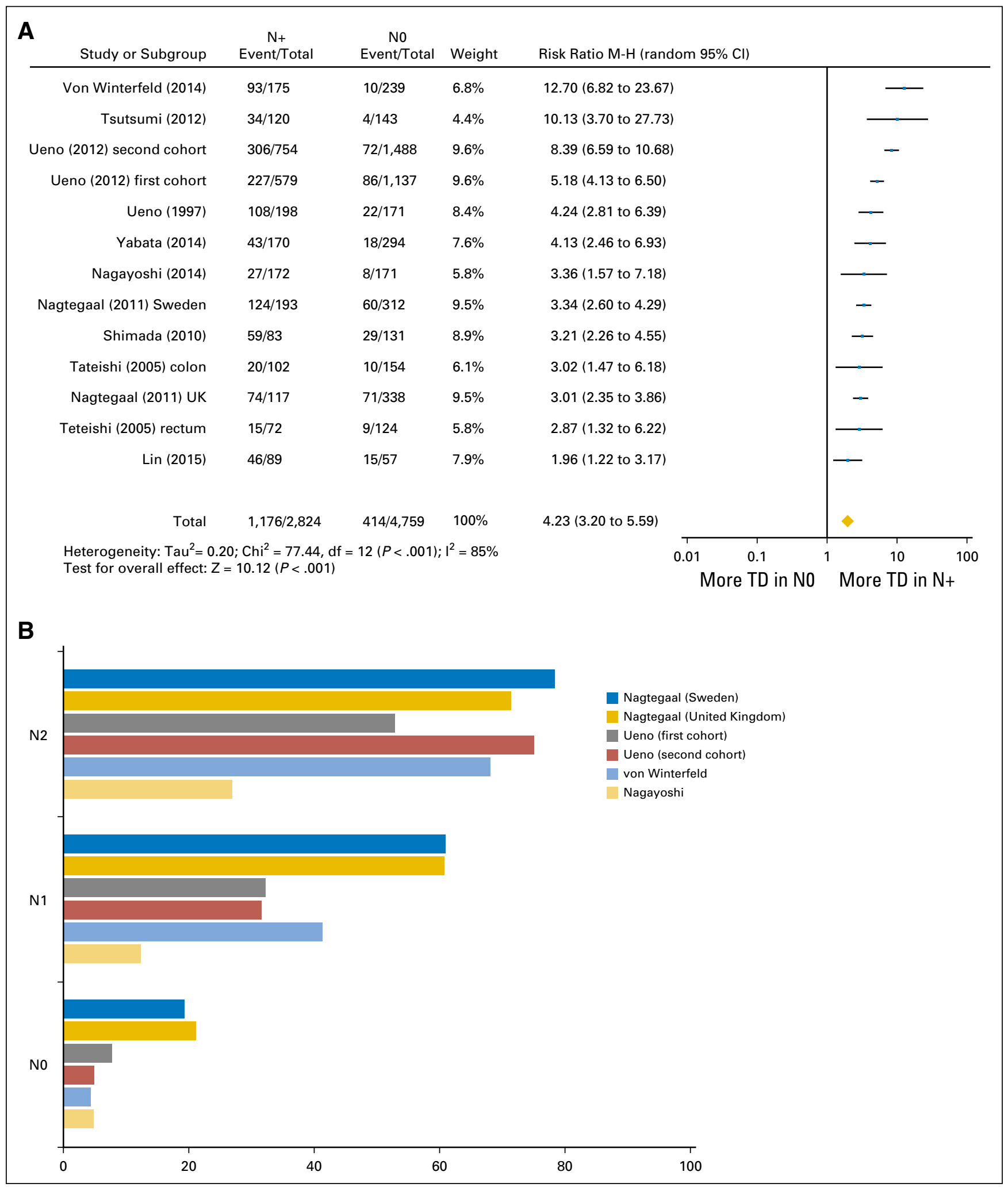

Fig A2. (A) The frequency of tumor deposits (TDs) in relation to $\mathrm{N}$ stage. (B) Risk ratio for TDs in relation to nodal status. (C) Risk ratio for TDs in relation to the presence of extramural vascular invasion (EMVI). EMVI-, no EMVI; EMVI+, EMVI present; NO, no lymph node metastases; N+, lymph node metastases present; TD-, TD negative; TD+, TD positive. 


\begin{tabular}{|c|c|c|c|c|c|c|c|}
\hline Study or Subgroup & $\begin{array}{c}\text { EMVI+ } \\
\text { Event/Total }\end{array}$ & $\begin{array}{c}\text { EMVI- } \\
\text { Event/Total }\end{array}$ & Weight & \multicolumn{4}{|c|}{ Risk Ratio $\mathrm{M}-\mathrm{H}$ (random 95\% Cl) } \\
\hline Tateishi (2005) rectum & $24 / 143$ & $0 / 53$ & $1.6 \%$ & \multicolumn{2}{|l|}{18.38 (1.14 to 296.91$)$} & & \\
\hline Tateishi (2005) colon & $29 / 184$ & $1 / 72$ & $2.9 \%$ & \multicolumn{2}{|l|}{11.35 (1.58 to 81.76$)$} & & \\
\hline Shimada (2010) & $87 / 196$ & $1 / 18$ & $3.1 \%$ & \multicolumn{2}{|l|}{7.99 (1.18 to 54.02$)$} & & \\
\hline Yabata (2014) & $58 / 334$ & $3 / 130$ & $6.8 \%$ & \multicolumn{2}{|l|}{7.52 (2.40 to 23.59 ) } & & \\
\hline Nagtegaal (2011) UK & $95 / 157$ & $50 / 298$ & $18.5 \%$ & \multicolumn{2}{|l|}{3.61 (2.72 to 4.78$)$} & $\rightarrow-$ & \\
\hline Nagtegaal (2011) Sweden & $43 / 59$ & $141 / 446$ & $19.5 \%$ & \multicolumn{2}{|l|}{2.31 (1.87 to 2.84$)$} & - & \\
\hline Puppa (2007) & $44 / 69$ & $56 / 159$ & $18.6 \%$ & \multicolumn{2}{|l|}{1.81 (1.37 to 2.39$)$} & - & \\
\hline Nagayoshi (2014) & $18 / 136$ & $16 / 205$ & $12.7 \%$ & \multicolumn{2}{|l|}{$1.70(0.90$ to 3.21$)$} & & \\
\hline Lin (2015) & $14 / 25$ & $47 / 121$ & $16.4 \%$ & \multicolumn{2}{|l|}{1.44 (0.95 to 2.18$)$} & - & \\
\hline Total & $412 / 1,303$ & $315 / 1,502$ & $100 \%$ & \multicolumn{2}{|l|}{2.60 (1.81 to 3.73$)$} & $\diamond$ & \\
\hline \multirow{2}{*}{\multicolumn{4}{|c|}{$\begin{array}{l}\text { Heterogeneity: } \mathrm{Tau}^{2}=0.16 ; \mathrm{Chi}^{2}=35.17, \mathrm{df}=8(P<.001) ;\left.\right|^{2}=77 \% \\
\text { Test for overall effect: } Z=5.16(P<.001)\end{array}$}} & 0.01 & 0.1 & $1 \quad 10$ & 100 \\
\hline & & & & \multicolumn{4}{|c|}{ More TD in EMVI- More TD in EVMI+ } \\
\hline
\end{tabular}

Fig A2. (Continued). 


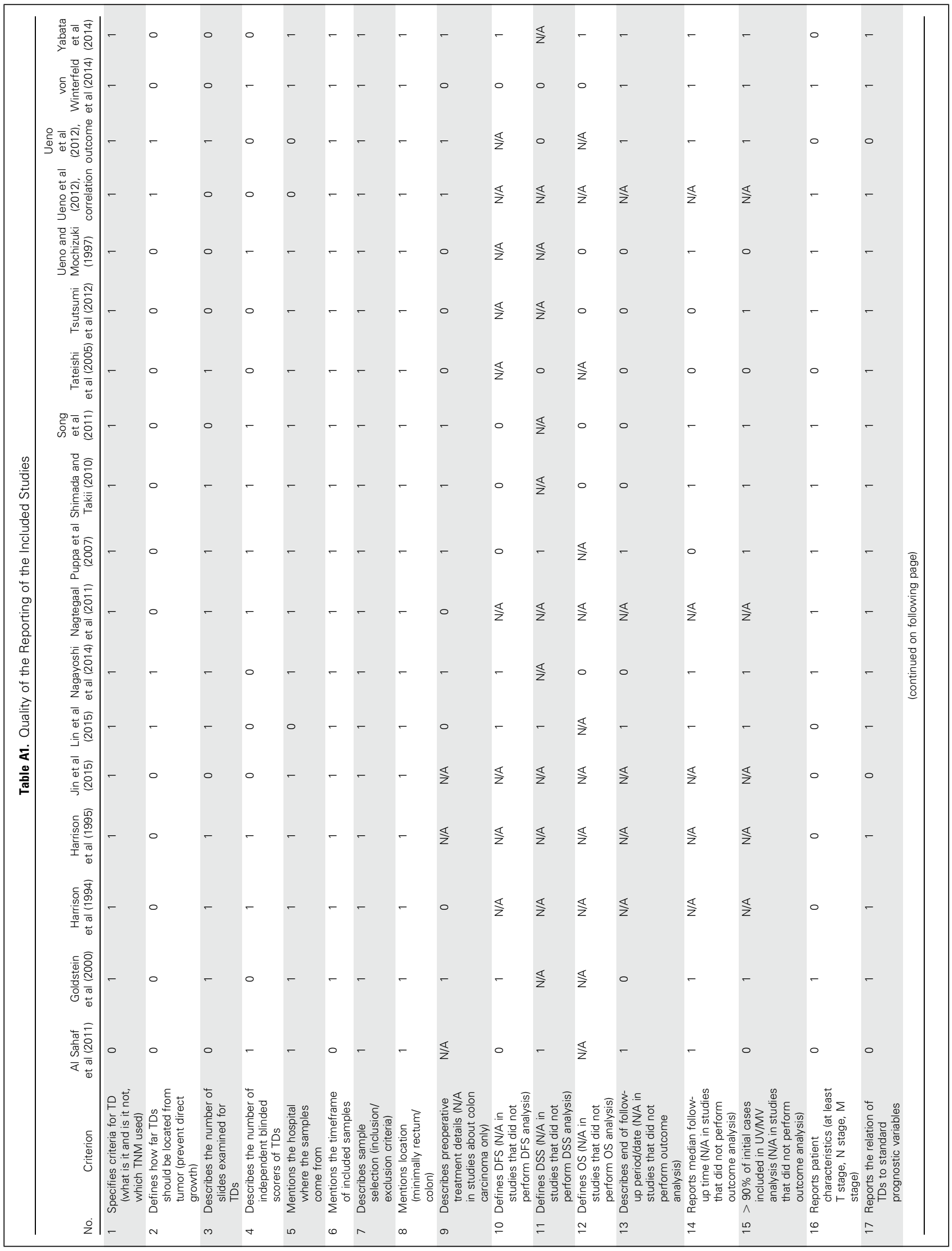




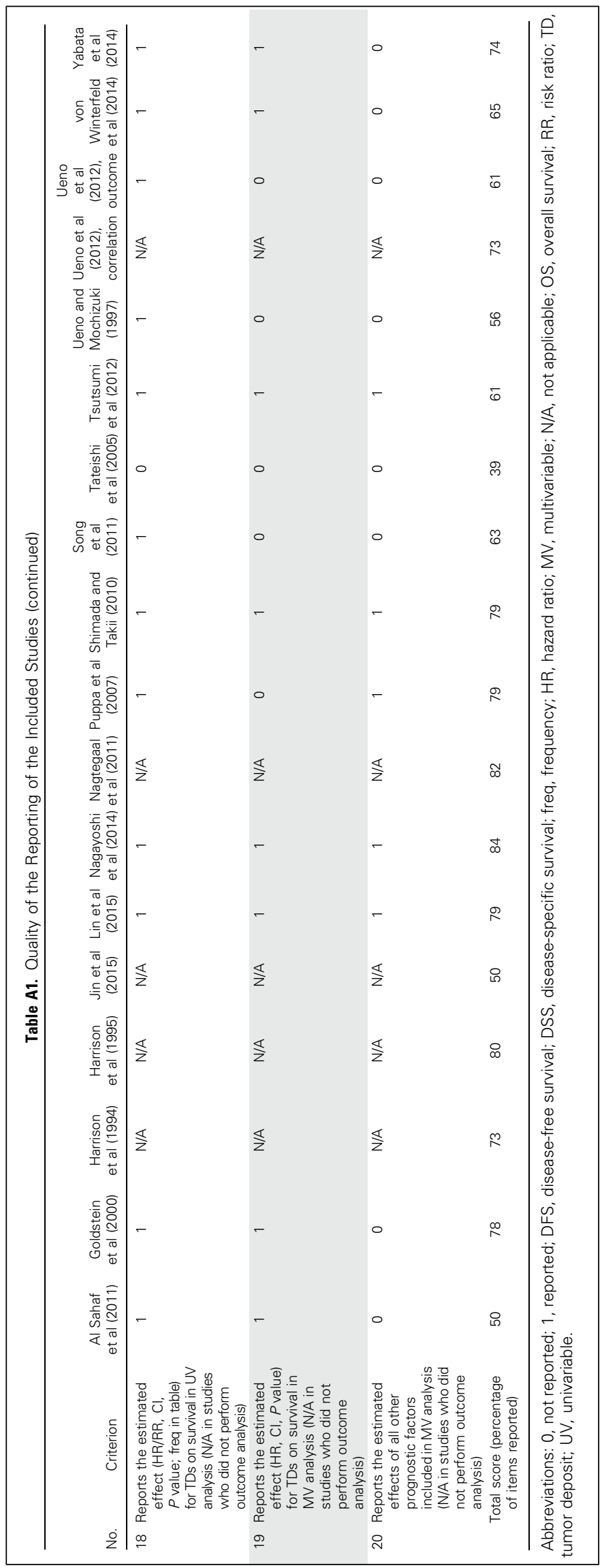


Nagtegaal et al

Table A2. Covariates That Were Included in Multivariable Analyses for Disease-Free Survival, Disease-Specific Survival, and Overall Survival per Included Study

\begin{tabular}{|c|c|c|c|c|c|c|}
\hline Study & pT & Lymph Node & TD & Tumor Characteristic & Treatment & Other \\
\hline \multicolumn{7}{|l|}{ Disease-free survival } \\
\hline Shimada and Takii & pT & $\mathrm{pN}^{*}$ & $T D^{*}$ & Grade, LVI, size & & \\
\hline Nagayoshi et al & pT & $\mathrm{pN}^{*}$ & $\mathrm{TD}^{*}$ & Grade* $^{*}$, LVI, VI & $\mathrm{ChT}^{*}$, margins & \\
\hline Lin et al & & $\mathrm{pN}$ & $\mathrm{TD}^{*}$ & & & $\begin{array}{l}\text { Gender, } \mathrm{LM}^{*} \text {, extrahepatic } \\
\text { metastases }\end{array}$ \\
\hline Goldstein et al & & LNM $^{*}$ & TD* & Grade*, LVI, VI, location & & Age \\
\hline Von Winterfeld et al & & & TD & Grade, location & ChT & Gender, age, TNM \\
\hline \multicolumn{7}{|l|}{ Disease-specific survival } \\
\hline Al Sahaf et al & pT & LN ratio* $\mathrm{EC}^{*}, \mathrm{~N}+$ & TD* & Grade, LVI & $\mathrm{ChT}^{*}$ & Gender, age \\
\hline Song et al & $p T^{*}$ & $\mathrm{pN}^{*}$ & $\mathrm{TD}^{*}$ & $L V I^{*}$ & & \\
\hline Von Winterfeld et al & & & $T D^{*}$ & Grade, location & ChT & Gender, age, TNM \\
\hline Lin et al & & $\mathrm{pN}^{*}$ & $\mathrm{TD}^{*}$ & $\mathrm{VI}^{*}, \mathrm{PNI}$ & & CEA, LM* \\
\hline \multicolumn{7}{|l|}{ Overall survival } \\
\hline Shimada and Takii & pT & $\mathrm{pN}$ & TD* & Grade, LVI, size* & & \\
\hline Yabata et al & $\mathrm{pT}^{*}$ & LNM & $\mathrm{TD}^{*}$ & $\begin{array}{l}\mathrm{LVI}{ }^{*}, \mathrm{VI} \text {, circumferential } \\
\text { occupancy }\end{array}$ & & $\mathrm{Age}^{*}$ \\
\hline Nagayoshi et al & $\mathrm{pT}$ & $\mathrm{pN}^{*}$ & TD* & Grade, LVI, VI & $\mathrm{ChT}^{*}$, margins* & \\
\hline Tsutsumi et al & $p T^{*}$ & $\mathrm{pN}$ & TD* & & & TNM \\
\hline Von Winterfeld et al & & & $\mathrm{TD}^{*}$ & Grade, location & $\mathrm{ChT}$ & Gender, age, TNM \\
\hline
\end{tabular}

Abbreviations: CEA, blood levels of carcinoembryonic antigen: ChT, adjuvant chemotherapy; EC, extracapsular growth of positive lymph nodes; LM, number of liver metastases; LNM, lymph node metastases; LN, lymph node; LVI, lymphatic invasion; PNI, perineural growth: TD, tumor deposit; VI, vascular invasion.

* Statistically significant $(P<.05)$. 